\title{
Construção de um equipamento de baixo custo para registro de biopotenciais cardíacos visando à análise da variabilidade da frequência cardíaca
}

Construction of low-cost equipment for recording cardiac biopotentials, aiming to analysis of heart rate variability

\author{
Aurélio de Brito Teixeira ${ }^{1,2^{*}}$, Rafael Pereira ${ }^{1,3}$
}

\begin{abstract}
Resumo
Os registros dos potenciais bioelétricos do coração são datados há mais de um século, e atualmente são utilizados tanto no contexto da clínica para instituição de diagnósticos, quanto para ensino e pesquisa nas áreas das ciências médicas. O objetivo desse estudo foi construir um equipamento de baixo custo, capaz de registrar e salvar em computador os dados de biopotenciais cardíacos para análise da variabilidade da frequência cardíaca (VFC), com possibilidade de registro com taxa de amostragem de $250 \mathrm{~Hz}$ a $2500 \mathrm{~Hz}$, o que viabilizaria o uso para fins didáticos e de pesquisa científica dos biopotenciais cardíacos, de humanos e de animais, por um mesmo equipamento de forma não invasiva. $\mathrm{O}$ equipamento proposto foi desenvolvido inicialmente em uma placa de prototipação universal a qual abarcou o sistema de aquisição, filtros e amplificação dos sinais bioelétricos do coração, que foram observados em um osciloscópio digital. A posteriori, foi desenvolvida uma versão final do protótipo como uma interface de ligação feita entre a placa do sistema e o computador através da placa e plataforma de software e hardware Arduino ${ }^{\circledR}$, onde foi possível salvar os dados e realizar as análises dos biopotenciais, inclusive da variabilidade da frequência cardíaca, com software Origin ${ }^{\circledR}$ e Kubios $\mathrm{HRV}^{\circledR}$. Os registros realizados com as taxas de amostragem propostas mostraram boa resolução temporal e viabilizaram a análise da VFC com boa coerência. Espera-se que o equipamento possa ser utilizado como facilitador em estudos que necessitem a captação de biopotenciais do coração em humanos e animais.
\end{abstract}

Palavras Chave: Eletrocardiografia. Tecnologia de Baixo Custo. Desenho de Equipamento. Controle Autonômico do Coração

\begin{abstract}
Recordings of the heart's electrical potentials are dated since the last century, and are currently used in the context of clinical diagnostic institution, as well as for teaching and research in the biomedical science areas. This study aimed to build a low-cost equipment, able to record and save to computer data from the heart's electrical potentials from human and animals with the same equipment, through the possibility of recording with sampling rate of $250 \mathrm{~Hz}$ to $2500 \mathrm{~Hz}$, which would be used for teaching and scientific research purposes. The proposed equipment was initially developed on a universal protoboard which encompassed the signal acquisition system, filters and amplification of the heart's bioelectric signals, that were observed in a digital oscilloscope. Subsequently, a final version of the prototype was developed to connect the signal acquisition system interface and the computer across a board and software platform and hardware Arduino® to save the data. The analysis of biopotentials, including the heart rate variability, was done with the softwares Origin ${ }^{\circledR}$ and Kubios HRV ${ }^{\circledR}$. Our results showed that recordings performed with the proposed sampling rates exhibited a good temporal resolution and enabled the analysis of HRV with good consistency. It is expected that the equipment can be used as a facilitator in studies that require the heart's biopotentials recordings from human and animal heart's.
\end{abstract}

Keywords: Electrocardiography. Low Cost Technology. Equipment Design. Heart Autonomic Control.

\section{Introdução}

O registro dos potenciais bioelétricos do coração em indivíduos vivos foi descrito há mais de cem anos por Willem Einthoven, sendo popularizado com a alcunha de Eletrocardiograma (ECG) (REIS et al., 2013). Boa parte de sua popularização deveu-se ao fato da necessidade de um equipamento de custo relativamente baixo para construção e

\footnotetext{
$\overline{\bar{*}}$ Aurélio de Brito Teixeira

aureliobrito@uesb.edu.br

1 Grupo de Pesquisa em Fisiologia Neuromuscular, Departamento de Ciências Biológicas, Universidade Estadual do Sudoeste da Bahia, Campus Jequié - Bahia, Brasil.

2 Acadêmico de Medicina, Departamento de Saúde II, Universidade Estadual do Sudoeste da Bahia, Campus Jequié - Bahia, Brasil.

${ }^{3}$ Docente do curso de Graduação em Medicina, Universidade Estadual do Sudoeste da Bahia, Campus Jequié - Bahia, Brasil.
}

manutenção, mas sua característica não invasiva e sua elevada sensibilidade para o diagnóstico de diversas doenças, tanto no ambiente ambulatorial como em unidades de urgência e emergência, o levaram a ser um dos principais exames complementares na prática clínica diária (LEE et al., 2008; WANG et al., 2012; REIS et al., 2013) .

O eletrocardiógrafo funciona como um galvanômetro medindo a diferença de potencial entre dois pontos através da utilização de eletrodos para captação dos sinais elétricos provenientes do músculo cardíaco que variam de -90 milivolts $(\mathrm{mV})$ a $20 \mathrm{mV}$ e chegam à superfície da pele em tensão máxima de cerca $1 \mathrm{mV}$ devido a resistência elétrica dos tecidos (SILVERTHORN, 2010; GUYTON e HALL, 2011; REIS et al., 2013). As ondas do ECG são 
representadas pelas letras $\mathrm{P}, \mathrm{Q}, \mathrm{R}, \mathrm{S}, \mathrm{T}, \mathrm{U}$, sendo o conjunto das ondas QRS a representação gráfica da despolarização ventricular, justificando a interpretação de que o intervalo entre dois picos de onda $\mathrm{R}$ (i.e., intervalo R-R) representa o intervalo de tempo entre dois batimentos cardíacos. A quantidade de picos R, por sua vez, medidos em um minuto, representa a frequência cardíaca do indivíduo (LEE et al., 2008; SILVERTHORN, 2010; GUYTON e HALL, 2011; WANG et al., 2012; REIS et al., 2013).

$\mathrm{O}$ registro das variações dos intervalos $\mathrm{R}-\mathrm{R}$ consecutivos permite a análise da variabilidade da frequência cardíaca, o que tem sido amplamente utilizado como ferramenta para estudo do controle autonômico do coração (MALIK et al., 1996; AUBERT et al., 2003; RAJENDRA et al., 2006; VANDERLEI et al, 2009). Sendo assim, a análise da VFC corresponde a um método não invasivo que permite a identificação do controle autonômico do coração em diversas situações, como em respostas a estímulos estressores, alterações do metabolismo, uso de drogas, sono, patologias e outros (MALIK et al., 1996; AUBERT et al., 2003; RAJENDRA et al., 2006; VANDERLEI et al., 2009).

Por convenção, a análise da VFC deve ser procedida com registros de no mínimo cinco minutos, o que representaria aproximadamente 350 intervalos R-R sucessivos a uma frequência cardíaca de 70 batimentos por minuto (BPM). Desta forma, percebe-se que a análise seria muito demorada e pouco precisa, se realizada através de registro em papel milimetrado. Por essa razão, as análises são realizadas com equipamentos que armazenam os dados do ECG. No entanto, nem todos eletrocardiógrafos disponíveis no mercado trazem a possibilidade de se ter os dados arquivados em um computador para análise, e os que viabilizam essa possibilidade apresentam um custo superior à maioria dos equipamentos convencionais por demandarem maiores recursos tecnológicos de hardware e software.

Outra limitação, inclusive dos equipamentos que armazenam os dados em computador, diz respeito às taxas de amostragem utilizadas, visto que, em sua maioria, há uma limitação de 250 Hertz (Hz), o que para utilização em humanos considera-se adequado para que se haja uma reconstituição do sinal sem perdas. Porém, para registros de ECG em animais, como camundongos, onde a frequência cardíaca chega a 600 BPM, a taxa de amostragem do sinal considerada adequada seria dez vezes superior, i.e., da ordem de $2500 \mathrm{~Hz}$.

Todavia, no mercado nacional, não se encontram facilmente equipamentos com essas características disponíveis, sendo em sua grande maioria adquiridos no exterior, o que impacta em altos custos de aquisição e importação. Sendo assim, o presente trabalho tem por objetivo construir um equipamento de baixo custo capaz de registrar e salvar em computador os dados de biopotenciais cardíacos para análise da variabilidade da frequência cardíaca, com possibilidade de registro com taxa de amostragem de $250 \mathrm{~Hz}$ a $2500 \mathrm{~Hz}$, o que viabilizaria o uso para fins didáticos e de pesquisa científica, dos biopotenciais cardíacos de humanos e de animais, por um mesmo equipamento, para análise da VFC de forma não invasiva.

\section{Material e métodos}

A descrição das etapas da construção do equipamento e técnica de registro dos biopotenciais cardíacos será apresentada em quatro subseções: 1) "sistema de aquisição dos biopotenciais", que descreve as características dos componentes eletrônicos utilizados para a aquisição/filtragem/amplificação dos biopotenciais; 2) "préprocessamento e armazenamento dos dados", que envolve a interface de comunicação com o computador e algoritmo de captação dos dados com conversão do sinal analógico para digital e armazenamento dos mesmos; 3) "alimentação do sistema e frequência de trabalho", que relata a descrição das fontes de alimentação de energia dos componentes do equipamento, bem como a frequência de trabalho do mesmo; 4) "registro $e$ análise dos registros dos biopotenciais cardíacos", que detalha a técnica de posicionamento dos eletrodos e os parâmetros analisados dos biopetenciais.

\section{Sistema de aquisição dos biopotenciais}

O sistema proposto nesse trabalho funciona na faixa de frequência de monitoramento, que é de $0,5 \mathrm{~Hz}$ a $50 \mathrm{~Hz}$, tendo em vista a recomendação desta faixa de frequência para fins de monitoramento ambulatorial, onde distúrbios do ritmo cardíaco, e não alterações morfológicas sutis no traçado do ECG, são o maior interesse (TOMPKINS, 2000). Adicionalmente, o uso desta faixa de frequência atenua ruídos de alta frequência oriundos da contração de músculos estriados esqueléticos (i.e., ruído eletromiográfico), assim como ruídos de baixa frequência oriundos de pequenos deslocamentos dos eletrodos em contato com a pele (TOMPKINS, 2000).

Para o sistema de aquisição foi utilizado um amplificador de instrumentação da Texas Instrument $^{\circledR}$, modelo INA128, indicado para obtenção de biopotenciais. O amplificador de instrumentação funciona amplificando a diferença de tensão entre os pinos 2 e 3 , que representam as entradas dos eletrodos do circuito e mantém alta impedância de entrada e rejeição de sinais de modo comum (TEXAS INSTRUMENT, 2015). A figura 1 apresenta um diagrama esquemático do circuito do INA128 e a equação para definição do ganho do circuito. De maneira sucinta, a amplificação nesse circuito é dada pela resistência de ganho $\left(R_{G}\right)$ ligada entre os pinos 1 e 8 , sendo definido para essa etapa do equipamento um ganho de 120 vezes e calculada a resistência para esse ganho $(\mathrm{G})$, conforme demonstrado na equação da figura 1. A saída do circuito é representada pelo pino 6 e os demais pinos são da alimentação simétrica. 


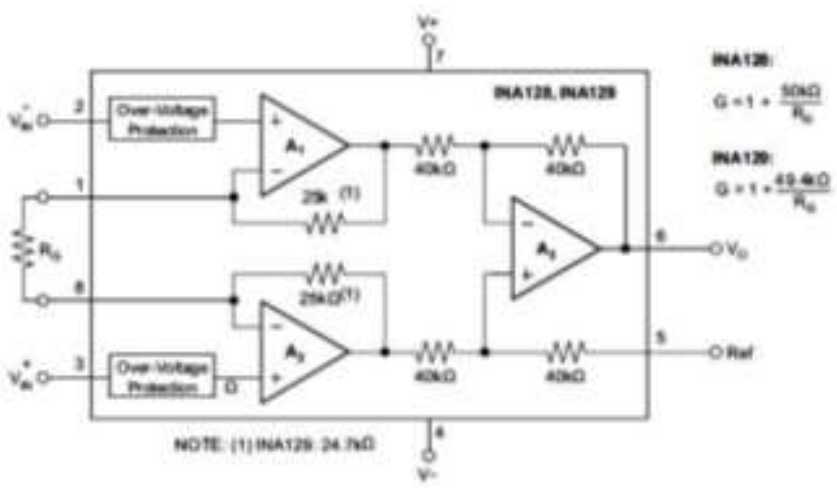

Figura 1. Esquemático INA128/129.

Na saída do circuito INA 128 foi implementado um filtro passivo passa alta (FPA) de $0,5 \mathrm{HZ}$, composto por capacitor e resistor, e a esse sinal foi implementado um filtro passa baixa ativo (FPB) de $50 \mathrm{~Hz}$ e ganho de 22 vezes composto por um amplificador operacional de um Circuito Integrado TL072, capacitor e resistor. O cálculo para frequência de corte dos filtros é determinado pela equação descrita abaixo, onde tem-se a frequência $(f)$, o valor do resistor $(\mathrm{R})$ utilizado e a capacitância (C) do capacitor:

$$
f=1 /(2 \text {. } \pi . \text { R. C) }
$$

Foi realizado um ajuste de nível em corrente contínua para que a saída do sinal se enquadrasse na faixa de operação da placa de prototipagem eletrônica Arduino ${ }^{\circledR}$ (descrito a diante) de $0-5 \mathrm{~V}$, assim foi definido através de um circuito de resistores e um potenciômetro ligados as tensões positiva e negativa da fonte a injeção de uma tensão regulável para ajuste da faixa de saída desejada.

O método de aterramento utilizado foi o Driven-RightLeg (DRL), que consiste em um circuito de realimentação, para aumentar a rejeição do modo comum (CMRR) do sistema a partir da divisão do resistor $\mathrm{R}_{\mathbf{G}}$ do ganho do INA128 em dois outros resistores com metade da resistência do primeiro. A interseção dos dois resistores representa a tensão do modo comum que é então invertida, amplificada e re-injetada na perna direita do indivíduo. Para essa etapa foi utilizado um circuito integrado TL072. A figura 2 apresenta o modelo de implementação do circuito com o amplificador de instrumentação, os filtros e o DRL.

\section{Pré-processamento e armazenamento dos dados}

A placa de prototipagem eletrônica open-source Arduino ${ }^{\circledR}$ Uno R3 foi utilizada para comunicação entre o sistema de aquisição e a comunicação com o computador. Trata-se de uma placa única com micro controlador, tendo ainda um conjunto de entradas/saídas embutidas e um software próprio. A comunicação com o computador é feita através de uma porta USB e um conversor Serial/USB embutido. As entradas analógicas da placa utilizada por padrão conseguem captar tensões de 0-5 Volts (V) em 1024 intervalos, i.e., a cada variação de $5 / 1024 \mathrm{~V}$ é possível fazer um registro diferente.

Para a etapa de implementação do algoritmo foi utilizado o ambiente de desenvolvimento integrado (IDE) Arduino ${ }^{\circledR}$, que consiste em um compilador que utiliza interface gráfica em Java baseado no ambiente Processing e constituído de um compilador gcc ( $\mathrm{C}$ e $\mathrm{C}++)$ baseado em Wiring. Por se tratar de uma plataforma open-source, inúmeras bibliotecas do sistema são encontradas gratuitamente na internet. $\mathrm{Na}$ implementação desse projeto foi utilizado um algoritmo que permite a leitura de uma das portas analógicas do Arduíno ${ }^{\circledR}$ e transmissão para um computador através da comunicação Serial/USB em taxas de amostragem configuráveis de $250 \mathrm{~Hz}$ a $2500 \mathrm{~Hz}$, modificando-se o valor numérico função Timer.initialize que representa o tempo em microssegundos que cada novo valor da saída analógica do equipamento deve ser capturado, conferindo-se assim um valor de 4000 para uma taxa de amostragens dos dados de $250 \mathrm{~Hz}$, e 400 para taxa de amostragem de $2500 \mathrm{~Hz}$ conforme demonstrado na figura 3. Os dados são plotados em tela, copiados e salvos como arquivo de texto através da utilização de qualquer software de edição de textos.

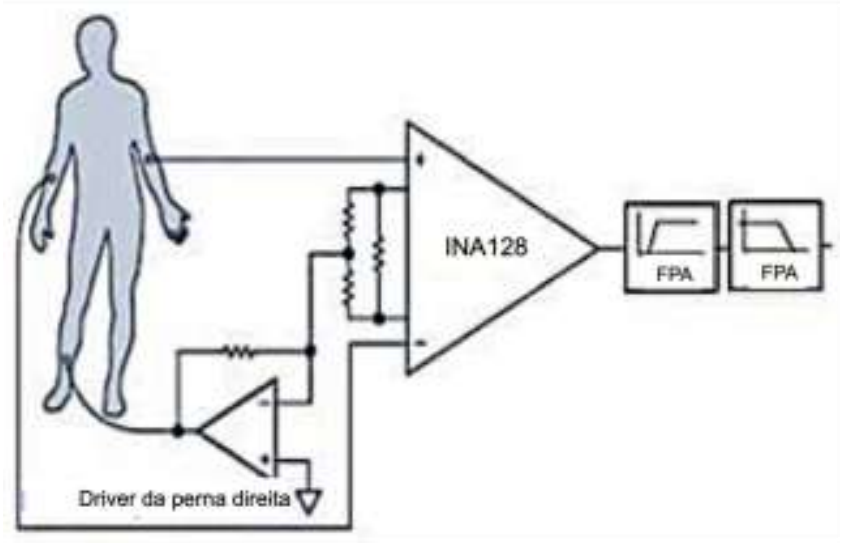

Figura 2: Modelo de representação do circuito de aquisição.

\section{Alimentação do sistema}

Para alimentação do circuito de aquisição dos sinais foi construída uma fonte simétrica adequada para funcionamento do amplificador de instrumentação e demais componentes a partir de duas baterias de $7,2 \mathrm{~V}$ ligadas em série, onde a intercessão entre as baterias define a referência $0 \mathrm{~V}$, tendo em uma das extremidades $+7,2 \mathrm{~V}$ e na outra $-7,2 \mathrm{~V}$. Posteriormente foram utilizados reguladores de tensão de $5 \mathrm{~V}$ bem como filtros tendo a saída final da fonte a seguinte configuração: $-5 \mathrm{~V}, 0 \mathrm{~V},+5 \mathrm{~V}$. A alimentação da placa Arduino é feita pela própria porta USB quando conectada a um computador.

\section{Registro e análise dos biopotenciais cardíacos}

Foram realizados dois registros de 5 minutos dos biopotenciais cardíacos com os eletrodos posicionados a 
nível do $5^{\circ}$ espaço intercostal na linha hemiclavicular dos lados direito e esquerdo, enquanto o eletrodo de referência foi posicionado próximo à crista ilíaca anterior direita, devido ao sistema DRL de aterramento adotado. Um dos registros foi realizado com taxa de amostragem de $250 \mathrm{~Hz}$, enquanto o outro com $2500 \mathrm{~Hz}$. Os registros foram coletados em um dos autores do trabalho.

Os dados armazenados foram submetidos um filtro passa-baixa com frequência de corte de $40 \mathrm{~Hz}$ no software OriginPRO $^{\circledR}$ V.8.5, visando suavizar o sinal sem perda de informações dos eventos cardíacos. Em seguida, os dados suavizados foram submetidos à identificação e extração dos intervalos RR sucessivos no software $\operatorname{Kubios}^{\circledR} \mathrm{HRV} 2.1$ (TARVAINEN et al., 2014). Da análise dos intervalos RR sucessivos foram extraídos parâmetros obtidos no domínio do tempo e da frequência.

Dentre os parâmetros obtidos no domínio do tempo foram calculados: a média dos intervalos RR registrados (Mean RR); raiz quadrada da média do quadrado das diferenças entre intervalos $\mathrm{RR}$ adjacentes (RMSSD); porcentagem dos intervalos $\mathrm{RR}$ adjacentes com diferença de duração maior que 50ms (pNN50); Índice Triangular, calculado dividindo-se a área corresponde ao histograma formado número total de intervalos $\mathrm{RR}$ utilizados construção do histograma e a altura, corresponde ao número de intervalos RR com frequência modal do triângulo; a interpolação triangular dos intervalos RR (TINN).

A análise no domínio da frequência foi realizada com a aplicação da Transformada Rápida de Fourier (FFT) com janelas de 256 s e sobreposição de $50 \%$, onde foram obtidos os seguintes parâmetros: magnitude normalizada dos componentes de baixa frequência (low-frequency power (LF) - $0.04-0.15 \mathrm{~Hz}$ ) do espectro e de alta frequência (high-frequency power (HF) - $0.15-0.4 \mathrm{~Hz}$ ). É reportado na literatura que a banda $\mathrm{HF}$ representa as oscilações do espectro influenciadas pelo sistema nervoso parassimpático, enquanto a banda LF representa as oscilações influenciadas predominantemente pelo sistema nervoso simpático, sendo a razão LF/HF um indicador de balanço simpatovagal (MALIK et al., 1996).

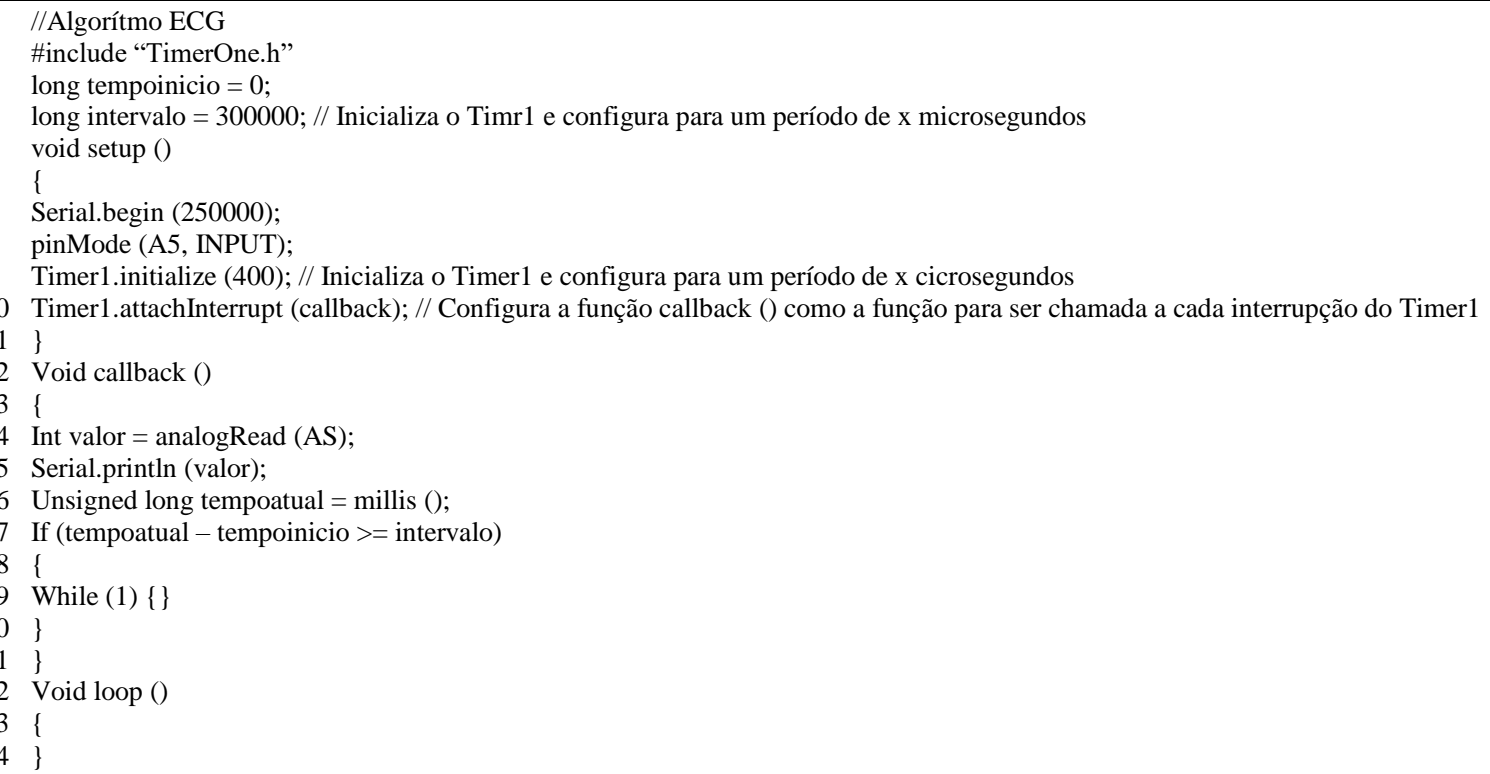

Figura 3: Algorítimo implementado na plataforma de desenvolvimento Arduino ${ }^{\circledR}$.

\section{Resultados}

Inicialmente, o equipamento proposto foi implementado em uma protoboard, pela facilidade de montagem, teste e modificação de componentes. Porém, uma série de efeitos adversos da mesma foram notados sobre o circuito, vide o mau contato entre a protoboard e componentes, proporcionando interferências e efeitos de capacitância parasita gerada entre as placas metálicas da placa de ensaio. Excluindo as dificuldades citadas anteriormente, essa etapa foi de suma importância para definição de um circuito final funcional que foi construído em etapa posterior.
A implementação do circuito final foi feita a partir de uma placa de fenolite padrão e acondicionados junto com a placa Arduino ${ }^{\circledR}$ e baterias em uma caixa de PVC. Conectores para recarga das baterias, saída de sinal analógico do circuito e conexão dos eletrodos, foram instalados. A figura 4 apresenta o protótipo desenvolvido na protoboard e a versão final do protótipo aqui desenvolvido.

Nos testes, utilizando a saída analógica do sistema de aquisição ligada a um osciloscópio digital, foi possível verificar os sinais do ECG com as ondas $\mathrm{P}$, o complexo QRS e a onda $\mathrm{T}$ conforme demonstrado na figura 5. No teste de captação dos dados através do algoritmo 
desenvolvido no IDE Arduino ${ }^{\circledR}$, foram executadas rotinas de amostragens dos dados em $250 \mathrm{~Hz}$ e $2500 \mathrm{~Hz}$ em forma de coordenadas do eixo vertical, através do serial monitor do IDE. Os registros foram analisados off-line (i.e., após o término dos registros) como descrito anteriormente.

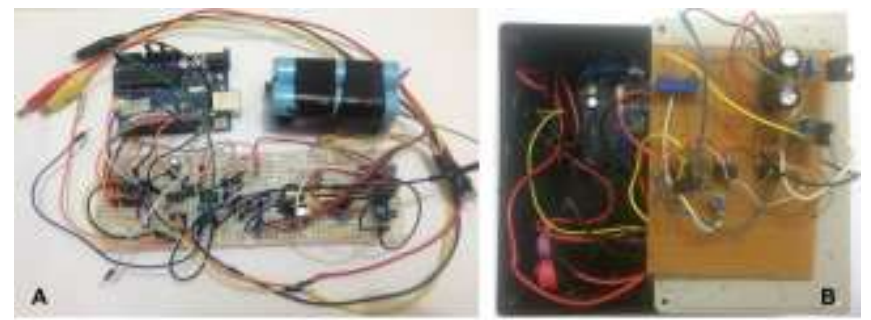

Figura 4. (A) Circuito montado em protoboard; (B) Versão final do protótipo.

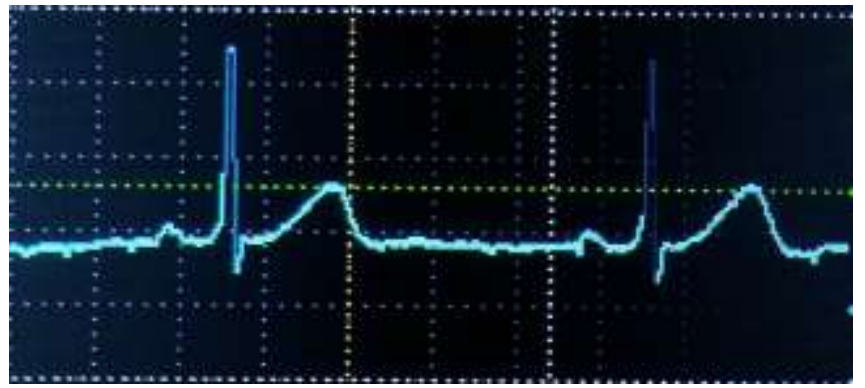

Figura 5: Sinal captado por osciloscópio digital ligado a saída analógica do circuito.

$\mathrm{Na}$ figura 6 são apresentados 10 segundos de registro dos biopotenciais cardíacos obtidos com taxa de amostragem de $250 \mathrm{~Hz}$ (A) e $2500 \mathrm{~Hz}$ (C), bem como a representação gráfica da variabilidade dos intervalos $\mathrm{RR}$ sucessivos obtidos durante os 5 minutos de registro com taxa de amostragem de $250 \mathrm{~Hz}$ (B) e $2500 \mathrm{~Hz}$ (D). É possível visualizar traçados típicos de eletrocardiograma de monitoramento, sendo as ondas $\mathrm{P}, \mathrm{Q}, \mathrm{R}, \mathrm{S}$ e $\mathrm{T}$ facilmente identificadas em A e C.

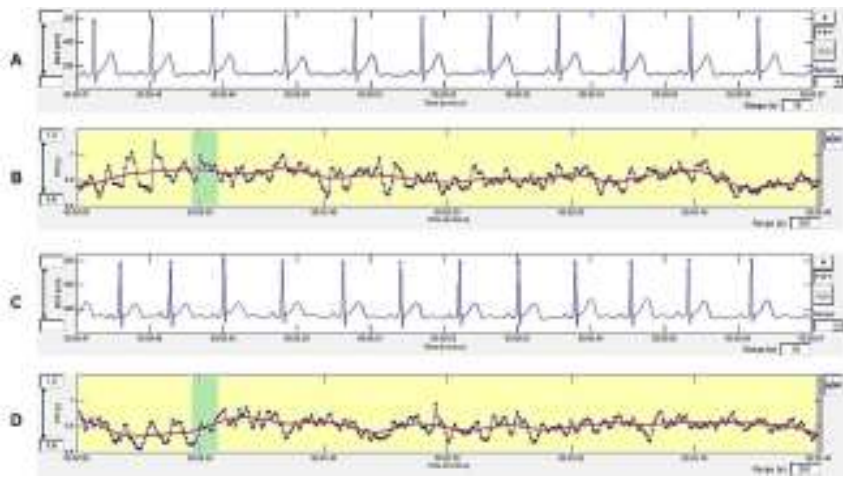

Figura 6: Representação gráfica de 10 segundos de registro dos biopotenciais cardíacos obtidos com taxa de amostragem de $250 \mathrm{~Hz}$ (A) e $2500 \mathrm{~Hz}$ (C). Representação gráfica da variabilidade dos intervalos RR sucessivos obtidos durante 5 minutos com taxa de amostragem de $250 \mathrm{~Hz}$ (B) e $2500 \mathrm{~Hz}$ (D).
A análise da variabilidade da frequência cardíaca dos dados registrados com 250 e $2500 \mathrm{~Hz}$ é apresentada na Tabela 1. É possível verificar que os valores dos parâmetros temporais e espectrais são bastante próximos entre os dois registros, sendo o MeanRR também muito próximo entre os registros. O MeanRR é um parâmetro menos sensível, mas não menos importante por estar atrelado ao número de pontos $\mathrm{R}$ presentes nos registros, os quais não foram registrados simultaneamente, por isso não é de se esperar que tenham valores idênticos, mas sim próximos. A partir dos dados de MeanRR é possível inferir que a frequência cardíaca nos registros era de 74 e 75 BPM nos registros com taxa de amostragem de 250 e $2500 \mathrm{~Hz}$, respectivamente.

Tabela 1: Parâmetros da variabilidade da frequência cardíaca obtidos no domínio do tempo e no domínio da frequência de biopotenciais cardíacos adquiridos com taxa de amostragem de 250 e $2500 \mathrm{~Hz}$.

\begin{tabular}{cccccc}
\hline & \multicolumn{5}{c}{ Domínio do tempo } \\
\cline { 2 - 6 } & $\begin{array}{c}\text { Mean RR } \\
(\mathbf{m s})\end{array}$ & $\begin{array}{c}\text { RMSSD } \\
(\mathbf{m s})\end{array}$ & $\begin{array}{c}\text { pNN50 } \\
(\boldsymbol{\%})\end{array}$ & $\begin{array}{c}\text { Triangular } \\
\text { index }\end{array}$ & $\begin{array}{c}\text { TINN } \\
(\mathbf{m s})\end{array}$ \\
\hline $\mathbf{2 5 0 H z}$ & 819.00 & 44.82 & 18.35 & 12.62 & 295.00 \\
$\mathbf{2 5 0 0 H z}$ & 796.15 & 35.09 & 13.03 & 14.50 & 250.00 \\
\hline \multicolumn{5}{c}{ Domínio da frequência } \\
\hline \multicolumn{5}{c}{ HF n.u. } \\
$\mathbf{2 5 0 H z}$ & 75.52 & & 24.46 \\
$\mathbf{2 5 0 0 H z}$ & 68.79 & 31.05 \\
\hline
\end{tabular}

\section{Discussão}

A dificuldade em encontrar equipamentos versáteis, que se adequem a certa variedade de condições específicas para a realização de atividades de ensino e/ou pesquisa e que apresentem uma boa relação custo-benefício é uma realidade que limita o avançar da ciência, especialmente em países emergentes (STOJANOVIĆ et al., 2015). Ressaltando também períodos de limitações de recursos financeiros disponibilizados para o desenvolvimento científico-tecnológico, como observado nos dias atuais. $\mathrm{O}$ presente estudo demonstrou que é possível desenvolver um equipamento versátil, configurável a diferentes necessidades experimentais, e de custo relativamente baixo.

O fato de viabilizar a escolha da taxa de amostragem para coleta dos biopotenciais cardíacos possibilitará que o mesmo equipamento seja utilizado para registro e análise de dados coletados com humanos e também em camundongos, o que não é viável, até o momento, com os equipamentos comerciais disponíveis no mercado nacional. Considerando que humanos apresentam uma frequência cardíaca média de aproximadamente 70 BPM, o que contrasta com a frequência de, aproximadamente, 600 BPM dos 
camundongos, uma taxa de amostragem de 100 a $250 \mathrm{~Hz}$ é suficiente para registro em humanos, enquanto que, para camundongos, há a necessidade de uma taxa de amostragem de 2000 a $2500 \mathrm{~Hz}$ (CHU et al., 2001; THIREAU et al., 2008). O equipamento aqui desenvolvido foi capaz de apresentar boa resolução temporal com registros realizados com $250 \mathrm{~Hz}$ e $2500 \mathrm{~Hz}$. No entanto, ambos os registros foram realizados em humanos, visto que a realização em camundongos ainda depende de alguns aspectos éticos de pesquisa envolvendo animais.

Outro aspecto importante dos resultados, obtidos neste estudo, foi o baixo custo para construção do equipamento. Outro aspecto importante dos resultados obtidos neste estudo foi o baixo custo para construção do equipamento, o que, mesmo considerando os gastos com testes de diferentes componentes até se alcançar o melhor conjunto, não excedeu a R \$ 300,00, o que, para fins de estabelecimento de parâmetros, corresponde a apenas $34 \%$ do valor do salário mínimo vigente no Brasil. Não obstante, esse custo pode ainda ser reduzido através da busca por fornecedores de peças eletrônicas com melhores preços, ou pela compra de quantidades maiores de peças, no caso de uma produção em série.

$\mathrm{O}$ equipamento proposto e desenvolvido nesse trabalho atende às expectativas iniciais e a sua utilização viabilizará a obtenção de biopotenciais em estudos científicos futuros. Sua utilização, através da identificação dos intervalos R-R, por um dado período de tempo, viabilizará a análise da variabilidade da frequência cardíaca, bem como outros seguimentos do ECG e monitoramento da frequência cardíaca, o que confere um grande número de possibilidades de utilização voltadas à prática de ensino e pesquisa nas áreas das Ciências Biológicas e da Saúde.

A implementação de filtros digitais online (i.e., durante o registro dos biopotenciais) pode melhorar o sinal adquirido, eliminando ruídos remanescentes, configurando assim, uma etapa adicional às etapas propostas neste projeto, visto que vão além da construção do hardware, envolvendo a implementação de funções avançadas em softwares de registro e análise de sinais.

\section{Conclusão}

Diante das dificuldades, experiências e resultados obtidos até aqui, melhorias e novas funcionalidades para o equipamento foram pensadas para serem implementadas em uma etapa posterior, tais como: desenvolvimento de software próprio para salvar os dados e plotar gráficos em tempo real dos biopotenciais; acoplamento de um display de cristal líquido no próprio equipamento para monitoramento do traçado do ECG e dos batimentos cardíacos.

Por conseguinte, é esperado que esse trabalho possa contribuir para realização de pesquisas futuras envolvendo tanto humanos, quanto animais, facilitando a quantidade e qualidade de estudos e envolvendo a coleta e análise dos biopotenciais do coração. Adicionalmente, é esperado também que estimule a produção de novos equipamentos de custo acessível visando o ensino e a pesquisa na área biomédica.

\section{Referências}

RAJENDRA-ACHARYA， U.; PAUL-JOSEPH， K.; KANNATHAL, N.; LIM, C.M.; SURI, J.S. Heart rate variability: a review. Medical and biological engineering and computing, v. 44, n. 12, p. 1031-1051, 2006.

AUBERT, A.E.; SEPS, B.; BECKERS, F. Heart rate variability in athletes. Sports medicine, v. 33, n. 12, p. 889-919, 2003.

CHU, V.; OTERO, J.M.; LOPEZ, O.; MORGAN, J.P.; AMENDE, I.; HAMPTON, T.G. Method for noninvasively recording electrocardiograms in conscious mice. BMC Physiology, v. 1, n. 6, 2001. https://doi.org/10.1186/1472-6793-1-6

GUYTON, A.C.; HALL, J.E.; GUYTON, A.C. Tratado de fisiologia médica. 12.ed., Rio de Janeiro, Elsevier, 2011.

LEE, C.S.; WANG, M.H. Ontological fuzzy agent for electrocardiogram application. Expert Systems with Applications, v. 35, n. 3, p. 1223-1236, 2008.

MALIK, M. Heart rate variability. Standards of measurement, physiological interpretation, and clinical use. European Heart Journal, v. 17, n. 3, p. 354-381, 1996.

REIS, H.J.L.; GUIMARÃES, H.P.; ZAZULA, A.D.; VASQUE, R.G.; LOPES, R.D. ECG: Manual prático de eletrocardiograma. São Paulo, Editora Atheneu, 2013.

SILVERTHORN, D.U. Fisiologia humana: uma abordagem integrada. 7.ed., Porto Alegre, Editora Artmed, 2010.

STOJANOVIĆ, R; CAPLANOVA, A.; KOVACEVIC, Z.; NEMANJA, N.; BUNDALO, Z. Alternative approach to addressing infrastructure needs in biomedical engineering programs (Case of emerging economies). Folia Medica Facultatis Medicinae Universitatis Saraeviensis, v. 50, n. 1, p. 29-33, 2015.

TARVAINEN, M.P.; NISKANEN, J.P.; LIPONNEN, J.A.; RANTA-AHO, P.O.; KARJALAINEN, P.A. Kubios HRVheart rate variability analysis software. Computer Methods and Programs in Biomedicine, v. 113, n. 1, p. 210-220, 2014.

TEXAS INSTRUMENT. INA12x Precision, Low Power Instrumentation Amplifiers. Texas: Texas Instrument; 2015.

THIREAU, J.; ZHANG, B.L.; POISSON, D.; BABUTY, D. Heart rate variability in mice: a theoretical and practical guide. Experimental Physiology, v. 93, n. 1, p. 83-94, 2008.

TOMPKINS, W.J. Biomedical Digital Signal Processing: C-language Examples and Laboratory Experiments for the IBM PC, New Jersey, Prentice Hall, 1993. 
VANDERLEI, L.C.M.; PASTRE, C.M.; HOSHI, R.A.; CARVALHO, T.D.; GODOY, M.F. Noções básicas de variabilidade da frequência cardíaca e sua aplicabilidade clínica. Revista Brasileira de Cirurgia Cardiovascular, v. 24, n. 2, p. 205-217, 2009.
WANG, M.H.; LEE, C.S.; ACAMPORA, G.; LOIA, V. Electrocardiogram application based on heart rate variability ontology and fuzzy markup language. In: ECG Signal Processing, Classification and Interpretation. London, Springer, 2012, p. 155-178. 